\title{
Association of immune cell subsets with cardiac mechanics in the Multi-Ethnic Study of Atherosclerosis
}

Arjun Sinha, ${ }^{1,2}$ Adovich S. Rivera, ${ }^{2}$ Margaret F. Doyle, ${ }^{3}$ Colleen Sitlani, ${ }^{4,5}$ Alison Fohner, ${ }^{6}$ Sally A. Huber, ${ }^{3}$ Nels C. Olson, ${ }^{3}$ Joao A.C. Lima, ${ }^{7}$ Joseph A. Delaney, ${ }^{6,8}$ Matthew J. Feinstein,, ${ }^{1,2}$ Sanjiv J. Shah, ${ }^{1}$

Russel P. Tracy, ${ }^{3,9}$ and Bruce M. Psaty $4,5,6,10$

'Department of Medicine and 'Department of Preventive Medicine, Feinberg School of Medicine, Northwestern University, Chicago, Illinois, USA. ${ }^{3}$ Department of Pathology and Laboratory Medicine, The Robert Larner, M.D. College of Medicine, University of Vermont, Burlington, Vermont, USA. ${ }^{4}$ Department of Medicine, ${ }^{5}$ Cardiovascular Health Research Unit, and ${ }^{6}$ Department of Epidemiology, University of Washington, Seattle, Washington, USA. 'Divison of Cardiology, Department of Medicine, Johns Hopkins University, Baltimore, Maryland, USA. ${ }^{8}$ College of Pharmacy, University of Manitoba, Winnipeg, Manitoba, Canada. 'Department of Biochemistry, The Robert Larner, M.D. College of Medicine, University of Vermont, Burlington, Vermont, USA. ${ }^{10}$ Department of Health Services, School of Public Health, University of Washington, Seattle, Washington, USA.

BACKCROUND. Immunomodulatory therapy may help prevent heart failure (HF). Data on immune cells and myocardial remodeling in older adults with cardiovascular risk factors are limited.

METHODS. In the Multi-Ethnic Study of Atherosclerosis cohort, 869 adults had 19 peripheral immune cell subsets measured and underwent cardiac MRI during the baseline exam, of which $\mathbf{3 2 1}$ had assessment of left ventricular global circumferential strain (LV-GCS). We used linear regression with adjustment for demographics, cardiovascular risk factors, and cytomegalovirus serostatus to evaluate the cross-sectional association of immune cell subsets with left ventricular mass index (LVMI) and LV-CCS.

RESULTS. The average age of the cohort was $61.6 \pm 10.0$ years and $53 \%$ were women. Higher proportions of $\gamma / \delta$ T cells were associated with lower absolute (worse) LV-CCS $(-0.105 \%$ [95\% CI $-0.164 \%,-0.046 \%]$ per 1 SD higher proportion of $\gamma / \delta$ T cells, $P=0.0006$ ). This association remained significant after Bonferroni's correction. Higher proportions of classical monocytes were associated with worse absolute LV-CCS $(-0.04 \%[95 \% \mathrm{Cl}-0.07 \%, 0.00 \%]$ per 1 SD higher proportion of classical monocytes, $P=0.04)$. This did not meet significance after Bonferroni's correction. There were no other significant associations with LV-GCS or LVMI.

CONCLUSION. Pathways associated with $\gamma / \delta$ T cells may be potential targets for immunomodulatory therapy targeted at HF prevention in populations at risk.

FUNDING. Contracts 75N92020D00001, HHSN268201500003I, N01-HC-95159, 75N92020D00005, N01HC-95160, 75N92020D00002, NO1-HC-95161, 75N92020D00003, N01-HC-95162, 75N92020D00006, N01-HC-95163, 75N92020D00004, N01-HC-95164, 75N92020D00007, N01-HC-95165, N01-HC-95166, N01-HC-95167, N01-HC-95168, and N01-HC-95169 and grant R01 HL98077 from the National Heart, Lung, and Blood Institute/NIH and grants KL2TR001424, UL1-TR-000040, UL1-TR-001079, and UL1TR-001420 from the National Center for Advancing Translational Sciences/NIH.

Copyright: () 2021, Sinha et al. This is an open access article published under the terms of the Creative Commons Attribution 4.0 International License.

Submitted: March 1, 2021

Accepted: May 27, 2021

Published: July 8, 2021

Reference information: /CI Insight. 2021;6(13):e149193.

https://doi.org/10.1172/jci.

insight.149193.

\section{Introduction}

In adults without heart failure (HF), higher levels of circulating proinflammatory cytokines, such as TNF- $\alpha$, IL-6, and C-reactive protein, have been associated with future HF events (1-3). However, targeting these inflammatory cytokines in adults who have developed HF has not been successful in slowing disease progression $(4,5)$. Secondary analysis of the Canakinumab Anti-Inflammatory Thrombosis Outcome Study showed a significant trend toward decreased HF events with IL-1 $\beta$ blockade in patients without HF but with 
prior myocardial infarction (MI; ref. 6). Thus, immunomodulatory therapies may be better suited for HF prevention in individuals at risk for $\mathrm{HF}$ and/or with subclinical cardiac mechanical impairment. Investigating the associations of innate and adaptive immune cells, integral in regulating the inflammatory milieu, with cardiac structure and function in older adults with cardiovascular risk factors but without HF can provide important insights into potential immune targets for HF prevention. In this study, we leveraged data obtained from a case-cohort substudy of the Multi-Ethnic Study of Atherosclerosis (MESA) evaluating peripheral blood mononuclear cells (PBMCs) and MI to investigate associations of innate and adaptive immune cell subsets with measures of cardiac structure and function on cardiac magnetic resonance imaging (CMR).

Due to limited data in humans without clinical disease, we examined a broad set of immune cell subsets. Nevertheless, our a priori hypotheses were guided by findings from experimental models of HF and clinical studies in humans with HF or broad cardiovascular disease (CVD). Proliferation and recruitment of monocytes from the circulation mediate cardiac hypertrophy and systolic dysfunction in mouse models (7-13). In humans, monocytes are categorized into classical, intermediate, and nonclassical subsets based on expression of CD14 (lipopolysaccharide) and CD16 (Fc $\mathrm{III})$ receptors. Classical monocytes (CD14 ${ }^{++} \mathrm{CD} 16^{-}$) express inflammatory cytokines while intermediate monocytes $\left(\mathrm{CD} 14^{++} \mathrm{CD} 16^{+}\right)$may have inflammation-regulating effects (14-16). Impacts of other innate immune cell subsets, such as natural killer (NK) and $\gamma / \delta$ T cells, on cardiac dysfunction have not been as thoroughly studied. NK cells are thought to have a regulatory function and may be protective while there is experimental evidence that $\gamma / \delta$ T cells promote myocardial inflammation (17-19).

The adaptive immune system also plays an important role in myocardial remodeling (20-22). T cells, broadly categorized into $\mathrm{CD}^{+}$and $\mathrm{CD}^{+} \mathrm{T}$ cells, can be subdivided into regulatory and effector subsets. $\mathrm{CD}^{+} \mathrm{T}$ regulatory cells normally have a beneficial modulatory effect but can become increasingly dys-

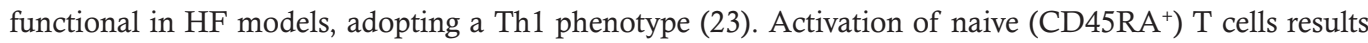
in clonal expansion with differentiation into effector subtypes, such as cytotoxic $\mathrm{CD} 8^{+} \mathrm{T}$ cells. For $\mathrm{CD} 4^{+} \mathrm{T}$ cells, effector subtypes can include proinflammatory T helper 1 (Th1) and T helper 17 (Th17) cells as well as profibrotic T helper 2 (Th2) cells. Each subset is elevated in circulation and in the myocardium of HF animal models $(24,25)$. In murine ischemic HF, there is polarization toward a Th2 and Th17 phenotype with reversal of cardiac hypertrophy and systolic dysfunction after inhibition of $\mathrm{CD}^{+} \mathrm{T}$ cells (24). Subsequently, these activated $\mathrm{T}$ cells give rise to a pool of antigen-specific memory $\left(\mathrm{CD} 45 \mathrm{RO}^{+}\right)$and terminally differentiated effector memory (TEMRA) T cells. Higher ratio of memory to naive T cells has been associated with atherosclerosis (26). With aging, there are also rising subpopulations of senescent (CD28-) T cells that are associated with comorbidities such as hypertension, diabetes, and atherosclerosis (27-30).

Given the consistent effects observed on cardiac hypertrophy and systolic function in experimental models, left ventricular mass index (LVMI) and left ventricular global circumferential strain (LV-GCS), a more sensitive measure of systolic function, were chosen as the primary outcomes. Both are also early imaging markers of cardiac remodeling and dysfunction that independently predict incident HF ( 31 , 32). Our a priori hypotheses, based on studies described above, were (a) higher circulating proportions of classical monocytes, $\gamma / \delta \mathrm{T}$ cells, and Th1 and Th17 cells are associated with worse cardiac structure and function (higher LVMI and lower absolute LV-GCS); and (b) higher circulating proportions of intermediate monocytes, NK cells, and regulatory $\mathrm{T}$ cells (Tregs) are associated with better cardiac structure and function (lower LVMI and higher absolute LV-GCS).

\section{Results}

Out of 1195 MESA participants who underwent immune cell phenotyping, 869 participants had CMR performed. The mean proportions of each immune cell subset are included in Supplemental Table 2; supplemental material available online with this article; https://doi.org/10.1172/jci.insight.149193DS1. The distributions of these cells were similar to those measured from fresh whole blood samples obtained during MESA Exam 4 (33). The baseline characteristics of these participants are included in Table 1 . The average age of the cohort was $61.6 \pm 10.0$ years with approximately 53\% women and 28\% Blacks. In addition, 321 participants also had tagged CMR for assessment of circumferential strain (Figure 1).

Our a priori hypotheses evaluated the associations of classical monocytes (CD14 $\left.{ }^{++} \mathrm{CD} 16^{-}\right)$, intermediate monocytes $\left(\mathrm{CD} 14^{+} \mathrm{CD} 16^{+}\right)$, and $\mathrm{NK}, \gamma / \delta$, Th1 $\left(\mathrm{CD} 4^{+} \mathrm{IFN}^{+}\right)$, Th17 $\left(\mathrm{CD} 4^{+} \mathrm{IL}-17^{+}\right)$, and regulatory $\left(\mathrm{CD} 4^{+} \mathrm{C}-\right.$ D25 $\left.{ }^{+} \mathrm{CD} 127^{-}\right) \mathrm{T}$ cells with LV-GCS and LVMI. Absolute strain values were used for ease of interpretation, with a lower absolute strain value representing less systolic deformation and worse function. As shown in Table 2, higher proportions of $\gamma / \delta \mathrm{T}$ cells were associated with lower absolute (worse) LV-GCS $(-0.11 \%$ 
Table 1. Weighted baseline characteristics of participants with cardiac MRI data from the case-cohort sample

\begin{tabular}{|c|c|c|}
\hline & $\begin{array}{l}\text { Cardiac MRI performed } \\
\qquad(n=869)\end{array}$ & $\begin{array}{l}\text { Circumferential strain assessed } \\
\qquad(n=321)\end{array}$ \\
\hline Age, years (mean \pm SD) & $61.6 \pm 10.0$ & $64.4 \pm 9.8$ \\
\hline Male, \% & 46.9 & 56.5 \\
\hline Female, \% & 53.1 & 43.5 \\
\hline \multicolumn{3}{|l|}{ Race/ethnicity, \% } \\
\hline White & 37.3 & 25.9 \\
\hline Chinese & 14.9 & 15.0 \\
\hline Black & 27.8 & 33.7 \\
\hline Hispanic & 20.0 & 25.4 \\
\hline \multicolumn{3}{|l|}{ Smoking, \% } \\
\hline Current & 11.6 & 10.0 \\
\hline Former & 35.2 & 35.2 \\
\hline Never & 53.2 & 54.8 \\
\hline $\mathrm{BMI}, \mathrm{kg} / \mathrm{m}^{2}($ mean $\pm \mathrm{SD})$ & $27.5 \pm 4.9$ & $27.8 \pm 4.4$ \\
\hline $\mathrm{SBP}, \mathrm{mmHg}($ mean $\pm \mathrm{SD})$ & $126.6 \pm 21.3$ & $128.2 \pm 19.9$ \\
\hline Diabetes, \% & 8.2 & 11.1 \\
\hline Statin use, $\%$ & 14.6 & 16.1 \\
\hline Total cholesterol, mg/dL (mean \pm SD) & $194.1 \pm 33.0$ & $192.8 \pm 32.5$ \\
\hline HDL-cholesterol, mg/dL (mean \pm SD) & $51.1 \pm 14.6$ & $49.3 \pm 14.1$ \\
\hline $\mathrm{eGFR}, \mathrm{mL} / \mathrm{min} / 1.73 \mathrm{~m}^{2}($ mean $\pm \mathrm{SD})$ & $74.5 \pm 16.1$ & $73.8 \pm 15.8$ \\
\hline Log-transformed CMV (mean \pm SD) & $4.0 \pm 2.4$ & $4.0 \pm 2.3$ \\
\hline Left ventricular EF (\%) & $57.0 \pm 6.0$ & $56.0 \pm 6.0$ \\
\hline Left ventricular mass index $\left(\mathrm{g} / \mathrm{m}^{2}\right)$ & $55.2 \pm 8.6$ & $55.6 \pm 8.5$ \\
\hline Left ventricular GCS (\%) & -- & $-17.0 \pm 2.2$ \\
\hline
\end{tabular}

Race/ethnicity was self-identified. SD, standard deviation; BMI, body mass index; SBP, systolic blood pressure; HDL, high-density lipoprotein; eGFR, estimated glomerular filtration rate; CMV, cytomegalovirus; EF, ejection fraction; GCS, global circumferential strain.

$[95 \% \mathrm{CI}-0.16 \%,-0.05 \%]$ per $1 \mathrm{SD}$ higher proportion of $\gamma / \delta \mathrm{T}$ cells, $P=0.0006)$. This association remained significant even after correction for multiple testing. Higher proportions of classical monocytes were associated with worse LV-GCS $(-0.04 \%[95 \%$ CI $-0.07 \%, 0.00 \%]$ per 1 SD higher proportion of classical monocytes, $P=0.04)$. However, this association was not significant after Bonferroni's correction. None of the other immune cell subsets selected a priori had a significant association with LV-GCS. There were also no significant associations among the remaining immune cell subsets and LV-GCS. Of note, the association of Th2 CD $4^{+} \mathrm{T}$ cells with LV-GCS $\left(0.25 \%\right.$ [95\% CI $-0.01 \%, 0.50 \%$ ] per $1 \mathrm{SD}$ higher proportion of Th2 CD4 ${ }^{+}$ T cells, $P=0.06$ ) had the largest effect size of all the immune cell subsets but did not meet statistical signifcance. As shown in Table 3, there were no significant associations between immune cell subsets and LVMI.

In addition, we performed prespecified analyses evaluating for interaction of immune cell subsets with sex and blood pressure with respect to LV-GCS. We observed a significant interaction between sex and classical monocytes but not $\gamma / \delta \mathrm{T}$ cells (Supplemental Table 3). In stratified analysis, higher proportions of classical monocytes were associated with worse LV-GCS $(-0.09 \%$ [95\% CI $-0.13 \%,-0.05 \%]$ per 1 SD higher proportion of classical monocytes, $P=0.0001$ ) in women (Table 4 ). There was no significant association observed between classical monocytes and LV-GCS in men. We further created estimated marginal plots to visualize the effect of sex on the associations between the immune cell subsets and LV-GCS. As illustrated in Supplemental Figure 6A, there was an inverse association between proportions of classical monocytes and LV-GCS in women while there was no significant association in men. Sex did not modify the association between $\gamma / \delta$ T cells and LV-GCS (Supplemental Figure $6 \mathrm{~B}$ ).

The associations of $\gamma / \delta$ T cells and classical monocytes with LV-GCS had significant interaction with hypertension status (Supplemental Table 4). In stratified analysis, higher proportions of $\gamma / \delta \mathrm{T}$ cells were associated with worse LV-GCS $(-0.17 \%[95 \% \mathrm{CI}-0.27 \%,-0.07 \%]$ per $1 \mathrm{SD}$ higher proportion of $\gamma / \delta \mathrm{T}$ cells, $P=0.0017$ ) in adults without hypertension (Table 5). Similarly, higher proportions of classical monocytes were associated with worse LV-GCS $(-0.10 \%[95 \%$ CI $-0.15 \%,-0.05 \%]$ per 1 SD higher proportion 


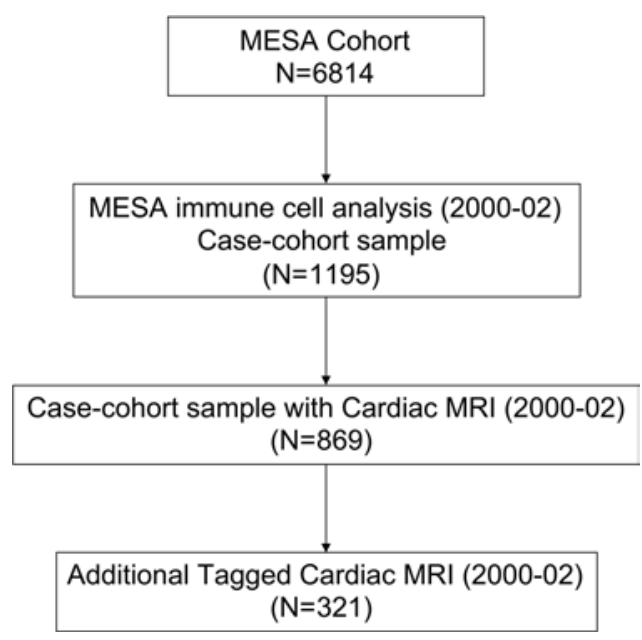

Figure 1. MESA study design with immune cell subset case-cohort and cardiac MRI.

of classical monocytes, $P=0.0003$ ) in adults without hypertension (Table 5). There was no significant association observed between $\gamma / \delta$ T cells or classical monocytes and LV-GCS in adults with hypertension. The estimated marginal plots further illustrate an inverse association of higher proportions of classical monocytes and $\gamma / \delta \mathrm{T}$ cells with LV-GCS in participants without hypertension but no clear association in those with hypertension (Supplemental Figure 7, A and B). In order to better understand the effect of antihypertensive medications, we further stratified the hypertensive group by treatment status (Table 5). In adults with untreated hypertension, higher proportions of $\gamma / \delta$ T cells were associated with worse LV-GCS $(-0.14 \%[95 \% \mathrm{CI}-0.26 \%,-0.02 \%]$ per $1 \mathrm{SD}$ higher proportion of $\gamma / \delta$ T cells, $P=0.04)$. But no association was observed between $\gamma / \delta$ T cells and LV-GCS in those on antihypertensive medications $(-0.04 \%$ [95\% CI $-0.10 \%, 0.03 \%$ ] per $1 \mathrm{SD}$ higher proportion of $\gamma / \delta \mathrm{T}$ cells, $P=0.28)$. In contrast, classical monocytes did not have an association with LV-GCS in adults with hypertension regardless of treatment status.

\section{Discussion}

In a multicenter cohort of middle-aged adults free of CVD, who had PBMCs collected and CMR performed during the same period, we observed a significant association between higher proportions of $\gamma / \delta$ $\mathrm{T}$ cells and worse cardiac function, as measured by absolute LV-GCS (Table 2). The association with $\gamma / \delta$ $\mathrm{T}$ cells was significantly modified by antihypertensive medication use (Table 5). While the association of higher proportions of classical monocytes with LV-GCS did not meet significance after Bonferroni's correction, there was a significant interaction (Tables 4 and 5) with sex and hypertension status. There was no significant association observed between any of the immune cell subsets and LVMI. These findings highlight specific immune cell subsets that may play a role in adverse cardiac remodeling in older adults with cardiovascular risk factors but without HF.

Our findings related to $\gamma / \delta$ T cells add to prior experimental results demonstrating the detrimental effects of $\gamma / \delta$ T cells in different types of cardiomyopathies. In our cohort, $\gamma / \delta$ T cells represented on average $6.7 \%$ of all $\mathrm{CD}^{+} \mathrm{T}$ cells in the peripheral blood. Unlike MHC-restricted $\alpha \beta \mathrm{CD} 4^{+} \mathrm{T}$ cells, a majority of $\gamma / \delta \mathrm{T}$ cells are activated in an MHC-independent manner by a broad set of antigens (34). The capacity of $\gamma / \delta \mathrm{T}$ cells to recognize antigens displayed by damaged tissue and rapidly respond within hours without requiring clonal expansion allows them to participate in immunosurveillance, referred to as lymphoid stress surveillance ( 35 , 36). Thus, $\gamma / \delta \mathrm{T}$ cells can perform a broad set of functions from creating a local effector response to triggering the adaptive immune system (36). Prior mechanistic studies have illustrated that $\gamma / \delta$ T cells promote infiltration of neutrophils and macrophages into the myocardium via pathways involving IL-17, IL-1 $\beta$, and upregulation of the pathogenic Th1 response $(18,37,38)$. We also observed that the association of $\gamma / \delta$ T cells with LV-GCS was significantly mitigated in individuals on antihypertensive medications, suggesting potential immunomodulatory effects. Comparisons of our findings with experimental models are inherently limited because immune cell subsets and pathways may markedly change when transitioning from a preclinical or subclinical state (captured by our cohort) to a disease state (captured by experimental models). Thus, our 
Table 2. Association of immune cell subsets with GCS

\begin{tabular}{|c|c|c|c|}
\hline Immune cell phenotype & Immune cell label & Estimate in \% (SEM) & $P$ value \\
\hline \multicolumn{4}{|l|}{ Innate immune cells } \\
\hline NK & Natural killer cells & $0.03(0.02)$ & 0.25 \\
\hline $\mathrm{CD}^{4} 4^{++} \mathrm{CD}_{16}^{-}$ & Classical monocytes & $-0.03(0.02)$ & 0.04 \\
\hline $\mathrm{CD}^{-} 4^{-} \mathrm{CD} 16^{++}$ & Nonclassical monocytes & $0.04(0.02)$ & 0.06 \\
\hline $\mathrm{CD}_{14}{ }^{+} \mathrm{CD}_{16}{ }^{+}$ & Intermediate monocytes & $0.02(0.02)$ & 0.32 \\
\hline $\mathrm{CD}^{+} \gamma \delta^{+}$ & $\gamma / \delta$ T cells & $-0.11(0.03)$ & 0.0006 \\
\hline \multicolumn{4}{|l|}{ CD4+ adaptive immune cells } \\
\hline $\mathrm{CD}^{+} \mathrm{IFN}^{+}$ & Th1 CD4 ${ }^{+} \mathrm{T}$ cells & $0.00(0.02)$ & 0.88 \\
\hline $\mathrm{CD}^{+} \mathrm{IL}^{+}$ & Th2 CD4+ $T$ cells & $0.25(0.13)$ & 0.06 \\
\hline $\mathrm{CD}^{+} \mathrm{IL}^{+} 7^{+}$ & Th17 CD4+ $\mathrm{T}$ cells & $0.10(0.19)$ & 0.61 \\
\hline $\mathrm{CD}^{+} \mathrm{CD}^{\circ} 8^{+}$ & Activated $\mathrm{CD}^{+} \mathrm{T}$ cells & $0.03(0.02)$ & 0.12 \\
\hline $\mathrm{CD}^{+}{ }^{+} \mathrm{CD}_{45 \mathrm{RA}^{+}}$ & CD4+ naive $T$ cells & $0.01(0.01)$ & 0.38 \\
\hline $\mathrm{CD}^{+}{ }^{+} \mathrm{CD} 28^{-}$ & Immunosenescent $\mathrm{CD} 4^{+} \mathrm{T}$ cells & $-0.01(0.02)$ & 0.72 \\
\hline $\mathrm{CD}^{+}{ }^{+} \mathrm{CD} 45 \mathrm{RO}^{+}$ & $\mathrm{CD}^{+}$memory T cells & $-0.02(0.01)$ & 0.10 \\
\hline $\mathrm{CD}^{+}{ }^{\mathrm{CD}} 25^{+} \mathrm{CD}_{127^{-}}$ & Tregs & $0.04(0.07)$ & 0.63 \\
\hline $\mathrm{CD}^{+}{ }^{+} \mathrm{CD} 28^{-} \mathrm{CD} 57^{+} \mathrm{CD} 45 \mathrm{RA}^{+}$ & CD4+ TEMRA cells & $0.03(0.03)$ & 0.38 \\
\hline \multicolumn{4}{|l|}{ CD8+ adaptive immune cells } \\
\hline $\mathrm{CD}^{+} \mathrm{CD}^{\circ} 8^{+}$ & Activated $\mathrm{CD}^{+} \mathrm{T}$ cells & $0.02(0.01)$ & 0.08 \\
\hline $\mathrm{CD}^{+}{ }^{+} \mathrm{CD} 45 \mathrm{RA}^{+}$ & $\mathrm{CD8}^{+}$naive $\mathrm{T}$ cells & $0.01(0.01)$ & 0.40 \\
\hline $\mathrm{CD}^{+}{ }^{+} \mathrm{CD} 28^{-}$ & Immunosenescent CD8+ $\mathrm{T}$ cells & $0.01(0.01)$ & 0.30 \\
\hline $\mathrm{CD}^{+}+\mathrm{CD}^{2} 5 \mathrm{RO}^{+} \mathrm{CD}^{+}$ & Memory T cells & $-0.02(0.02)$ & 0.19 \\
\hline 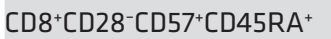 & CD8+ TEMRA cells & $0.02(0.01)$ & 0.21 \\
\hline
\end{tabular}

Immune cell subsets selected a priori are listed in red. Analysis adjusted for age, sex, race/ethnicity, smoking status, BMI, diabetes status, SBP, total cholesterol, HDL-cholesterol, statin use, eGFR, and log-transformed CMV serostatus. SEM, standard error of the mean; TEMRA, terminally differentiated memory effector.

results are hypothesis generating and can only be extrapolated to adults without clinical HF. Further studies are needed to determine if higher proportions of $\gamma / \delta \mathrm{T}$ cells reflect a state of dysregulated immunosurveillance that contributes to progressive adverse myocardial remodeling in adults with cardiovascular risk factors.

We also observed an inverse association between higher proportions of classical monocytes and LV-GCS that was significant at the $P$ value threshold of less than 0.05 but not after Bonferroni's correction. However, there was a strong interaction with sex, as there was a significant association between higher proportions of classical monocytes and LV-GCS in women but not in men (Supplemental Figure 6A). Classical monocytes are the predominant monocyte subtype in humans, making up roughly $85 \%$ of all circulating monocytes (39). They infiltrate injured tissue and differentiate into monocyte-derived macrophages and dendritic cells with the ability to either augment tissue damage or promote tissue regeneration and healing (40). Disturbing this delicate balance can alter the fate of the injured tissue. Experimental studies have demonstrated an association between classical monocytes and cardiac dysfunction in ischemic models (41, 42). In humans, peripheral monocytosis in adults with $\mathrm{MI}$ is associated with greater cardiac dysfunction, and adults with heart failure with preserved ejection fraction ( $\mathrm{HFpEF}$ ) also have higher levels of peripheral monocytes (43-46). However, it is important to note that our cohort did not include individuals with CVD, and thus the pathways regulating different immune cell subsets are likely different in our cohort compared with experimental models and cohorts, including individuals with existing CVD. There is evidence suggesting sex-related differences in monocytes. Monocyte levels increase with age in women but not in men, and animal models have demonstrated greater macrophage activation with phagocytic and inflammatory activity in female animals (47-50). While estrogen levels decrease after menopause, as would be expected in our relatively older cohort, there is evidence that sex-specific gene regulation can occur in the absence of circulating sex hormones (51). Thus, the relationship observed here between classical monocytes and worse cardiac function in women merits further investigation. Future studies should investigate if and how classical monocytes may play a role in progression from subclinical disease to clinical HF, especially $\mathrm{HFpEF}$, in women with cardiovascular risk factors, given the rising incidence of HFpEF in women (52).

Study limitations. Our study had important limitations. First, this is a cross-sectional study. Thus reverse causality cannot be ruled out, although it is unlikely given the individuals in the cohort did not have clinical 
Table 3. Association of immune cell subsets with LVMI

\begin{tabular}{|c|c|c|}
\hline Immune cell subset & Estimate in $\mathrm{g} / \mathrm{m}^{2}$ (SEM) & $P$ value \\
\hline \multicolumn{3}{|l|}{ Innate immune cells } \\
\hline NK cells & $-0.06(0.06)$ & 0.29 \\
\hline Classical monocytes & $0.00(0.38)$ & 0.94 \\
\hline Nonclassical monocytes & $-0.02(0.06)$ & 0.75 \\
\hline Intermediate monocytes & $0.01(0.05)$ & 0.78 \\
\hline$\gamma / \delta \mathrm{T}$ cells & $-0.04(0.05)$ & 0.39 \\
\hline \multicolumn{3}{|l|}{ CD4+ adaptive immune cells } \\
\hline Th1 CD4 ${ }^{+} \mathrm{T}$ cells & $0.03(0.04)$ & 0.53 \\
\hline Th2 CD4+ ${ }^{+}$cells & $-0.24(0.19)$ & 0.20 \\
\hline Th17 CD4+ $T$ cells & $-0.07(0.17)$ & 0.70 \\
\hline Activated $\mathrm{CD}^{+} \mathrm{T}$ cells & $-0.00(0.02)$ & 0.88 \\
\hline CD4+ Naive T cells & $0.02(0.03)$ & 0.43 \\
\hline Immunosenescent $\mathrm{CD} 4^{+} \mathrm{T}$ cells & $0.04(0.04)$ & 0.22 \\
\hline CD4+ memory T cells & $-0.01(0.02)$ & 0.58 \\
\hline Tregs & $-0.10(0.15)$ & 0.48 \\
\hline TEMRA cells & $0.02(0.07)$ & 0.78 \\
\hline \multicolumn{3}{|l|}{ CD8+ adaptive immune cells } \\
\hline Activated $\mathrm{CD}^{+}{ }^{+} \mathrm{T}$ cells & $-0.04(0.03)$ & 0.13 \\
\hline CD8 ${ }^{+}$naive $T$ cells & $-0.01(0.02)$ & 0.68 \\
\hline Immunosenescent CD8+ $\mathrm{T}$ cells & $-0.01(0.02)$ & 0.75 \\
\hline CD8+ memory T cells & $0.02(0.03)$ & 0.47 \\
\hline TEMRA cells & $-0.01(0.02)$ & 0.68 \\
\hline
\end{tabular}

Immune cell subsets selected a priori are listed in red. Analysis adjusted for age, sex, race/ethnicity, smoking status, BMI, diabetes status, SBP, total cholesterol, HDL-cholesterol, statin use, eGFR, and log-transformed CMV serostatus.

$\mathrm{HF}$ at the time of PBMC collection and CMR scanning. Future longitudinal studies are needed to account for intraindividual variability and understand the changes in immune cell subsets that occur over time with aging and accrual of cardiovascular comorbidities. Furthermore, better characterization of tissue-resident leukocytes is needed because peripheral immune cells may not be representative of myocardial resident immune cells, especially in the absence of an acute insult (53). Second, as with any observational study, there may be residual confounding, even though we adjusted for important potential confounders in our models. Third, only a subgroup of the participants with immune cell subset data underwent CMR, and a smaller subgroup received a tagged CMR study to evaluate for GCS. The limited sample size may have introduced selection bias and reduced power. However, the selection bias was likely minimal because the participants were largely chosen at random for the primary CMR and the tagged CMR studies. Last, participants were chosen for immune cell subset measurement using a case-cohort design, thus weighting the cohort toward individuals who went on to develop CVD. We adjusted for this by using sampling weights to minimize the bias of the estimates.

Conclusions. We observed a significant association between higher proportions of $\gamma / \delta$ T cells and worse cardiac function as measured by LV-GCS. This finding in an older population-based cohort with cardiovascular risk factors but without HF provides insight into which immune cell subsets may contribute to subclinical cardiac dysfunction even in the absence of acute myocardial injury. Our findings suggest that pathways associated with $\gamma / \delta \mathrm{T}$ cells and classical monocytes may be potential targets for immunomodulatory therapy targeted at HF prevention in similar populations with cardiovascular risk factors. Future mechanistic and longitudinal studies should evaluate the role of $\gamma / \delta$ T cells in development of cardiac dysfunction and $\mathrm{HF}$.

\section{Methods}

Study cohort. The MESA is a multicenter, population-based cohort comprising 6814 men and women aged 45 to 84 years at baseline, recruited from 6 field centers in the United States. Exclusion criteria included active treatment for cancer, amputation, pregnancy, or a history of clinical CVD, including MI, angina, cardiovascular procedures, HF, and/or cerebrovascular disease. Participants were interviewed and examined at the baseline exam (2000-2002). Participants in MESA self-identified as White, Black, Hispanic, or Chinese, and additional details on the design of the MESA cohort have been described previously (54). As part of a case (participants with coronary heart disease)-cohort study, immune cell phenotypes were obtained on 
Table 4. Association of classical monocytes with GCS stratified by sex

\begin{tabular}{ccc}
\hline Sex & Estimate in \% (SEM) & P value \\
Females & $-0.09(0.02)$ & 0.0001 \\
Males & $0.02(0.02)$ & 0.09
\end{tabular}

Analysis adjusted for age, race/ethnicity, smoking status, BMI, diabetes status, systolic blood pressure, total cholesterol, HDL-cholesterol, statin use, eGFR, and log-transformed CMV.

1195 MESA participants from PBMCs obtained and stored at baseline (55). For this study, we leveraged this immune cell phenotyping data to determine cross-sectional associations with cardiac structure and function obtained through CMR performed at baseline. Although a case-cohort design may reduce power for the primary question of association with MI, the design uses sampling weights to obtain valid estimates of associations with other phenotypes and thus provides opportunities for additional studies such as this one (56).

Clinical data. Clinical covariate data collected during the baseline exam in 2000 to 2002 were used. Standardized questionnaires were used to obtain demographics, past medical history, medications, and smoking status. Smoking was defined as never, former (no cigarettes within the past 30 days), or current. Trained clinical staff measured the weight, height, and resting blood pressure of each participant. Fasting lipid profile and serum creatinine were also obtained for each participant. IgG antibodies against CMV were measured by enzyme immunoassay (Diamedix Corp). The interassay coefficients of variation were $5.1 \%$ to $6.8 \%$ for the CMV IgG immunoassay.

Immune cell subset phenotyping. Immune cell phenotyping performed in this cohort has been previously described in detail $(55,57-59)$. PBMCs were isolated from blood collected in $8 \mathrm{~mL}$ citrate CPT tubes (BD Biosciences) during the MESA baseline exam in 2000 to 2002. The isolated PBMCs were washed and cryopreserved in media containing $90 \%$ fetal bovine serum and $10 \%$ dimethylsulfoxide at $-135^{\circ} \mathrm{C}$. At the time of phenotyping (2016), the cells were thawed at $37^{\circ} \mathrm{C}$ for 15 minutes and treated with benzonase (MilliporeSig$\mathrm{ma}, 250 \mathrm{U} / \mathrm{mL}$ in RPMI from Invitrogen, Thermo Fisher Scientific) for 20 minutes. Cells were slowly diluted 10 -fold by the addition of RPMI supplemented with fetal bovine serum (10\%), L-glutamine ( $2 \mathrm{mM})$, penicillin $(100 \mathrm{IU} / \mathrm{mL})$, and streptomycin $(100 \mu \mathrm{g} / \mathrm{mL})$ (fsRPMI) with mixing. Cells were pelleted by centrifugation $(5$ minutes at $200 \mathrm{~g}$ at room temperature) and treated with $250 \mathrm{U} / \mathrm{mL}$ benzonase in fsRPMI for 10 minutes. The pellet was washed and filtered through a $70 \mu \mathrm{m}$ filter.

For surface labeling, cells were incubated with LIVE/DEAD stain (Thermo Fisher Scientific catalog L34955 or L34957) for 15 minutes in PBS, $\mathrm{pH}$ 7.4. The viability stain was removed by centrifugation for 5 minutes at $200 \mathrm{~g}$ at room temperature, and antibodies or isotype-matched controls were added as detailed in Supplemental Table 1 and incubated for 15 minutes at room temperature in the dark. Excess antibody was removed by centrifugation, the cells were washed, and the final pellet was placed in $1 \%$ paraformaldehyde and stored refrigerated until flow cytometry was performed. All antibodies were from Miltenyi Biotec and used at manufacturer-recommended dilutions. Preliminary testing indicated that the inclusion of Fc block in this system did not significantly alter the percentages of the measured cell types, and so Fc block was not included.

Table 5. Association of immune cell subsets with GCS stratified by hypertension status

\begin{tabular}{cccc}
\hline Immune cell subset & Hypertension status & Estimate in \% (SEM) & P value \\
Classical monocytes & Normotensive & $-0.10(0.03)$ & 0.0003 \\
Classical monocytes & Hypertensive (treated) & $0.01(0.02)$ & 0.37 \\
Classical monocytes & Hypertensive (untreated) & $0.03(0.04)$ & 0.32 \\
$\gamma / \delta$ T cells & Normotensive & $-0.17(0.05)$ & 0.0017 \\
$\gamma / \delta$ T cells & Hypertensive (treated) & $-0.04(0.03)$ & 0.28 \\
$\gamma / \delta$ T cells & Hypertensive (untreated) & $-0.14(0.06)$ & 0.04
\end{tabular}

Analysis adjusted for age, race/ethnicity, smoking status, BMI, diabetes status, total cholesterol, HDL-cholesterol, statin use, eGFR, and log-transformed CMV serostatus. 
For intracellular staining assays (Th1, Th2, and Th17), PBMCs were activated with phorbol myristic acid/ionomycin in the presence of brefeldin A, as previously described (33). After incubating for 3 hours at $37^{\circ} \mathrm{C}, 5 \% \mathrm{CO}_{2}$, cells were centrifuged, resuspended in PBS at $\mathrm{pH} 7.4$, incubated with a LIVE/DEAD stain (Thermo Fisher Scientific catalog L34955) for 15 minutes at room temperature, and then centrifuged at $300 \mathrm{~g}$ for 5 minutes at room temperature. The PBMCs were resuspended in CD4/CD8 antibodies or appropriate isotypes and incubated for 15 minutes at room temperature in the dark. Samples were centrifuged and the pellets were washed and fixed with $2 \%$ paraformaldehyde (Alfa Aesar catalog 43368) for 10 minutes. Paraformaldehyde was removed by centrifugation for 5 minutes at $200 \mathrm{~g}$ at room temperature, and cells were incubated with antibodies against IL-4, IL-17, and IFN- $\gamma$ in the presence of $1 \%$ saponin (MilliporeSigma). After 15 minutes, cells were washed and resuspended in paraformaldehyde until flow cytometry was performed.

Flow cytometry was performed on an MQ10 flow cytometer and analyzed with MACS Quantify software (Miltenyi Biotec). Calibration beads were used for daily calibration. Compensation was set using single-color compensation controls, and isotype controls were used to set negative gates for each assay. Cell phenotypes were expressed as percentages. Flow cytometry gating strategies are presented in Supplemental Figures 1-5. All data are presented as percentages (CD4 subsets as $\% \mathrm{CD}^{+} \mathrm{T}$ cells, $\mathrm{CD} 8$ subsets as $\% \mathrm{CD} 8^{+} \mathrm{T}$ cells, monocyte subsets as $\% \mathrm{CD}_{14} 4^{+}$monocytes, $\gamma / \delta \mathrm{T}$ cells as $\% \mathrm{CD}^{+} \mathrm{T}$ cells, and NK cells as $\%$ gated lymphocytes). Percentages, instead of absolute numbers, were used since PBMCs for this cohort were cryopreserved for many years. Due to sample manipulation from cryopreservation, our internal analyses have demonstrated percentages, rather than absolute counts, to be a better representation of circulating cells in the whole blood.

Cardiac MRI. CMR was performed in 5098 participants during the MESA baseline exam in 2000 to 2002. Of these, 1773 individuals were randomly selected to undergo tagged CMR for LV-GCS measurement as an ancillary study protocol during the baseline exam. In our study sample, 869 of the 1195 participants included in the case-cohort sample underwent CMR, of whom 321 also had a tagged CMR. The 2 primary CMR outcomes were LVMI and LV-GCS. MRI data were acquired using 1.5 T scanners (General Electric and Siemens). The CMR protocol has been described previously $(60,61)$. Briefly, 2- and 4-chamber cine MRI series were acquired after standard scout imaging. Short-axis cine images were acquired from above the mitral valve to the left ventricular (LV) apex with retrospective gating. Three tagged short-axis slices from LV base to apex were obtained using an electrocardiographically triggered, segmented, $k$-space fast spoiled gradient-echo pulse sequence. The endocardial and epicardial borders were contoured using a semiautomated method (MASS 4.2, Medis). The difference between the epicardial and endocardial areas for each slice at end diastole was multiplied by the slice thickness, which was then multiplied by the density of the myocardium to determine the LV mass. Papillary muscle mass was excluded from the LV mass. LV mass was indexed to the body surface area. Short-axis-tagged slices were analyzed using the HARP method (MATLAB, MathWorks, or HARP1.15, Diagnosoft). Circumferential strain was calculated for 4 segments (anterior, posterior, lateral, and septal) from each of the 3 short-axis slices. LV-GCS describes circumferential shortening during systole and thus is a negative value, with more negative values denoting better function (62). LV-GCS had excellent intraobserver, interobserver, and interstudy reproducibility, with intraclass correlation coefficients ranging from 0.7 to 0.9 $(62,63)$. Segments with significant noise were excluded from the analysis (7.9\%). LV-GCS was calculated by averaging the existing segments. Absolute LV-GCS values are reported for ease of interpretation.

Statistics. Linear regression was used to determine the association between the proportions of immune cell subsets and the CMR parameters. Given the case-cohort design, sampling weights were incorporated into the regression model to obtain unbiased estimates, and robust SEM estimates were used. One model was used for each immune cell subset (independent variable) and CMR parameter (dependent variable). The models were adjusted for potential confounders, which included baseline age, sex, race/ethnicity, smoking status, BMI, diabetes status, SBP, total cholesterol, HDL-cholesterol, baseline statin use, eGFR, and log-transformed CMV serostatus. As part of our a priori analytic plan, we evaluated potential interactions with sex and hypertension, defined as SBP at least $140 \mathrm{mmHg}$ or use of antihypertensive medications, in immune cell subsets that had a significant association with either of the CMR outcomes. If the interaction term was significant, we performed stratified analyses and created estimated marginal plots to visually display how sex or hypertension modified the association between the immune cell subset and the CMR outcome. Bonferroni's correction was used to account for multiple testing. For the 7 immune cell subsets included in the a priori hypotheses, $P$ value threshold was set at $\leq 0.003$ (14 tests). For the remaining 12 immune cell subsets, $P$ value threshold was set at $\leq 0.001$ (38 total tests).

Study approval. All participants provided written informed consent for participation in the study, and all procedures were conducted under institutionally approved protocols for human subject research. The study protocol 
was approved by the IRBs of the coordinating center (University of Washington, Seattle, Washington, USA) and each of the MESA field centers.

\section{Author contributions}

AS designed the study and wrote the manuscript. ASR, CS, AF, and JAD analyzed the data and edited the manuscript. MFD, SAH, and NCO conducted the experiments and edited the manuscript. JACL acquired the data and edited the manuscript. MFD, SJS, RPT, and BMP designed the study and edited the manuscript.

\section{Acknowledgments}

The authors thank the other investigators, the staff, and the participants of the MESA for their valuable contributions. A full list of participating MESA investigators and institutions can be found at http://www. mesa-nhlbi.org. This research was supported by contracts 75N92020D00001, HHSN268201500003I, N01HC-95159, 75N92020D00005, N01-HC-95160, 75N92020D00002, N01-HC-95161, 75N92020D00003, N01HC-95162, 75N92020D00006, N01-HC-95163, 75N92020D00004, N01-HC-95164, 75N92020D00007, N01HC-95165, N01-HC-95166, N01-HC-95167, N01-HC-95168, and N01-HC-95169 and grant R01 HL98077 from the National Heart, Lung, and Blood Institute/NIH and by grants KL2TR001424, UL1-TR-000040, UL1-TR-001079, and UL1-TR-001420 from the National Center for Advancing Translational Sciences/NIH.

Address correspondence to: Arjun Sinha, Division of Cardiology, Department of Medicine, Department of Preventive Medicine, Northwestern University Feinberg School of Medicine, 680 N. Lake Shore Drive, Suite 1400, Chicago, Illinois 60611, USA. Phone: 916.432.0242; Email: arjun.sinha@northwestern.edu.

1. Vasan RS, et al. Inflammatory markers and risk of heart failure in elderly subjects without prior myocardial infarction: the Framingham Heart Study. Circulation. 2003;107(11):1486-1491.

2. Kalogeropoulos A, et al. Inflammatory markers and incident heart failure risk in older adults: the Health ABC (Health, Aging, and Body Composition) study. J Am Coll Cardiol. 2010;55(19):2129-2137.

3. Ohkuma T, et al. Cardiac stress and inflammatory markers as predictors of heart failure in patients with type 2 diabetes: the ADVANCE Trial. Diabetes Care. 2017;40(9):1203-1209.

4. Chung ES, et al. Randomized, double-blind, placebo-controlled, pilot trial of infliximab, a chimeric monoclonal antibody to tumor necrosis factor-alpha, in patients with moderate-to-severe heart failure: results of the anti-TNF Therapy Against Congestive Heart Failure (ATTACH) trial. Circulation. 2003;107(25):3133-3140.

5. Mann DL, et al. Targeted anticytokine therapy in patients with chronic heart failure: results of the Randomized Etanercept Worldwide Evaluation (RENEWAL). Circulation. 2004;109(13):1594-1602.

6. Everett BM, et al. Anti-inflammatory therapy with canakinumab for the prevention of hospitalization for heart failure. Circulation. 2019;139(10):1289-1299.

7. Sager HB, et al. Proliferation and recruitment contribute to myocardial macrophage expansion in chronic heart failure. Circ Res. 2016;119(7):853-864.

8. Bajpai G, et al. Tissue resident CCR2 - and CCR2+ cardiac macrophages differentially orchestrate monocyte recruitment and fate specification following myocardial injury. Circ Res. 2019;124(2):263-278.

9. Ismahil MA, et al. Remodeling of the mononuclear phagocyte network underlies chronic inflammation and disease progression in heart failure: critical importance of the cardiosplenic axis. Circ Res. 2014;114(2):266-282.

10. Dick SA, et al. Self-renewing resident cardiac macrophages limit adverse remodeling following myocardial infarction. Nat Immunol. 2019;20(1):29-39

11. Epelman S, et al. Embryonic and adult-derived resident cardiac macrophages are maintained through distinct mechanisms at steady state and during inflammation. Immunity. 2014;40(1):91-104.

12. Lavine $\mathrm{KJ}$, et al. Distinct macrophage lineages contribute to disparate patterns of cardiac recovery and remodeling in the neonatal and adult heart. Proc Natl Acad Sci U S A. 2014;111(45):16029-16034.

13. Molawi K, et al. Progressive replacement of embryo-derived cardiac macrophages with age. J Exp Med. 2014;211(11):2151-2158.

14. Wrigley BJ, et al. CD14++CD16+ monocytes in patients with acute ischaemic heart failure. Eur J Clin Invest. 2013;43(2):121-130.

15. Wrigley BJ, et al. Increased expression of cell adhesion molecule receptors on monocyte subsets in ischaemic heart failure. Thromb Haemost. 2013;110(1):92-100.

16. Wrigley BJ, et al. Increased formation of monocyte-platelet aggregates in ischemic heart failure. Circ Heart Fail. 2013;6(1):127-135.

17. Ong S, et al. Natural killer cells in inflammatory heart disease. Clin Immunol. 2017;175:26-33.

18. Huber S, et al. Gammadelta T cells promote a Th1 response during coxsackievirus B3 infection in vivo: role of Fas and Fas ligand. J Virol. 2002;76(13):6487-6494.

19. Huber SA. T cells expressing the gamma delta T cell receptor induce apoptosis in cardiac myocytes. Cardiovasc Res. 2000;45(3):579-587.

20. Nevers T, et al. Left ventricular T-cell recruitment contributes to the pathogenesis of heart failure. Circ Heart Fail. 2015;8(4):776-787.

21. Yndestad A, et al. Enhanced expression of inflammatory cytokines and activation markers in T-cells from patients with chronic heart failure. Cardiovasc Res. 2003;60(1):141-146.

22. Kallikourdis M, et al. T cell costimulation blockade blunts pressure overload-induced heart failure. Nat Commun. $2017 ; 8: 14680$. 
23. Bansal SS, et al. Dysfunctional and proinflammatory regulatory T-lymphocytes are essential for adverse cardiac remodeling in ischemic cardiomyopathy. Circulation. 2019;139(2):206-221.

24. Bansal SS, et al. Activated T lymphocytes are essential drivers of pathological remodeling in ischemic heart failure. Circ Heart Fail. 2017;10(3):e003688.

25. Fukunaga T, et al. Relation between CD4+ T-cell activation and severity of chronic heart failure secondary to ischemic or idiopathic dilated cardiomyopathy. Am J Cardiol. 2007;100(3):483-488.

26. Olson NC, et al. Decreased naive and increased memory CD4(+) T cells are associated with subclinical atherosclerosis: the multi-ethnic study of atherosclerosis. PLoS One. 2013;8(8):e71498.

27. Giubilato S, et al. Expansion of CD4+CD28null T-lymphocytes in diabetic patients: exploring new pathogenetic mechanisms of increased cardiovascular risk in diabetes mellitus. Eur Heart J. 2011;32(10):1214-1226.

28. Youn JC, et al. Immunosenescent CD8+ T cells and C-X-C chemokine receptor type 3 chemokines are increased in human hypertension. Hypertension. 2013;62(1):126-133.

29. Lee YH, et al. Senescent T cells predict the development of hyperglycemia in humans. Diabetes. 2019;68(1):156-162.

30. Bergström I, et al. Persistent accumulation of interferon- $\gamma$-producing CD8 + CD $56+\mathrm{T}$ cells in blood from patients with coronary artery disease. Atherosclerosis. 2012;224(2):515-520.

31. de Simone G, et al. Left ventricular mass predicts heart failure not related to previous myocardial infarction: the Cardiovascular Health Study. Eur Heart J. 2008;29(6):741-747.

32. Choi E-Y, et al. Prognostic value of myocardial circumferential strain for incident heart failure and cardiovascular events in asymptomatic individuals: the multi-ethnic study of atherosclerosis. Eur Heart J. 2013;34(30):2354-2361.

33. Tracy RP, et al. T-helper type 1 bias in healthy people is associated with cytomegalovirus serology and atherosclerosis: the Multi-Ethnic Study of Atherosclerosis. J Am Heart Assoc. 2013;2(3):e000117.

34. Lawand $\mathrm{M}$, et al. Key features of gamma-delta T-cell subsets in human diseases and their immunotherapeutic implications. Front Immunol. 2017;8:761.

35. Hayday AC. Gammadelta T cells and the lymphoid stress-surveillance response. Immunity. 2009;31(2):184-196.

36. Vantourout P, Hayday A. Six-of-the-best: unique contributions of $\gamma \delta$ T cells to immunology. Nat Rev Immunol. 2013;13(2):88-100.

37. Yan X, et al. Deleterious effect of the IL-23/IL-17A axis and $\gamma \delta$ T cells on left ventricular remodeling after myocardial infarction. $J$ Am Heart Assoc. 2012;1(5):e004408.

38. Li Y, et al. $\gamma \delta \mathrm{T}$ Cell-derived interleukin-17A via an interleukin-1 $\beta$-dependent mechanism mediates cardiac injury and fibrosis in hypertension. Hypertension. 2014;64(2):305-314.

39. Kapellos TS, et al. Human monocyte subsets and phenotypes in major chronic inflammatory diseases. Front Immunol. 2019;10:2035.

40. Hulsmans M, et al. Monocyte and macrophage contributions to cardiac remodeling. J Mol Cell Cardiol. 2016;93:149-155.

41. Dewald O, et al. CCL2/Monocyte chemoattractant protein-1 regulates inflammatory responses critical to healing myocardial infarcts. Circ Res. 2005;96(8):881-889.

42. Nahrendorf $\mathrm{M}$, et al. The healing myocardium sequentially mobilizes two monocyte subsets with divergent and complementary functions. J Exp Med. 2007;204(12):3037-3047.

43. Maekawa Y, et al. Prognostic significance of peripheral monocytosis after reperfused acute myocardial infarction:a possible role for left ventricular remodeling. J Am Coll Cardiol. 2002;39(2):241-246.

44. Hulsmans M, et al. Cardiac macrophages promote diastolic dysfunction. J Exp Med. 2018;215(2):423-440.

45. Glezeva N, et al. Exaggerated inflammation and monocytosis associate with diastolic dysfunction in heart failure with preserved ejection fraction: evidence of M2 macrophage activation in disease pathogenesis. J Card Fail. 2015;21(2):167-177.

46. DeBerge M, et al. Macrophages in heart failure with reduced versus preserved ejection fraction. Trends Mol Med. 2019;25(4):328-340.

47. Bongen E, et al. Sex differences in the blood transcriptome identify robust changes in immune cell proportions with aging and influenza infection. Cell Rep. 2019;29(7):1961-1973.

48. Klein SL, Flanagan KL. Sex differences in immune responses. Nat Rev Immunol. 2016;16(10):626-638.

49. Spitzer JA. Gender differences in some host defense mechanisms. Lupus. 1999;8(5):380-383.

50. Seillet $\mathrm{C}$, et al. The TLR-mediated response of plasmacytoid dendritic cells is positively regulated by estradiol in vivo through cell-intrinsic estrogen receptor $\alpha$ signaling. Blood. 2012;119(2):454-464.

51. Steegenga WT, et al. Sexually dimorphic characteristics of the small intestine and colon of prepubescent C57BL/6 mice. Biol Sex Differ. 2014;5:11.

52. Chang PP, et al. Trends in hospitalizations and survival of acute decompensated heart failure in four US communities (2005-2014): ARIC Study Community Surveillance. Circulation. 2018;138(1):12-24.

53. Bajpai G, et al. The human heart contains distinct macrophage subsets with divergent origins and functions. Nat Med. 2018;24(8):1234-1245

54. Bild DE, et al. Multi-ethnic study of atherosclerosis: objectives and design. Am J Epidemiol. 2002;156(9):871-881.

55. Olson NC, et al. Innate and adaptive immune cell subsets as risk factors for coronary heart disease in two population-based cohorts. Atherosclerosis. 2020;300:47-53.

56. Prentice RL. A case-cohort design for epidemiologic cohort studies and disease prevention trials. Biometrika. 1986;73(1):1-11.

57. Olson NC, et al. Associations of innate and adaptive immune cell subsets with incident type 2 diabetes risk: the MESA Study. J Clin Endocrinol Metab. 2020;105(3):848-857.

58. Delaney JAC, et al. Natural killer cells, gamma delta T cells and classical monocytes are associated with systolic blood pressure in the multi-ethnic study of atherosclerosis (MESA). BMC Cardiovasc Disord. 2021;21(1):45.

59. Feinstein MJ, et al. Nonclassical monocytes (CD14dimCD16+) are associated with carotid intima-media thickness progression for men but not women: the multi-ethnic study of atherosclerosis. Arterioscler Thromb Vasc Biol. 2021;41(5):1810-1817.

60. Castillo E, et al. Quantitative assessment of regional myocardial function with MR-tagging in a multi-center study: interobserver and intraobserver agreement of fast strain analysis with harmonic phase (HARP) MRI. J Cardiovasc Magn Reson. 2005;7(5):783-791.

61. Yoneyama K, et al. Natural history of myocardial function in an adult human population: serial longitudinal observations from MESA. JACC Cardiovasc Imaging. 2016;9(10):1164-1173. 
62. Donekal S, et al. Interstitial fibrosis, left ventricular remodeling, and myocardial mechanical behavior in a population-based multiethnic cohort: the multi-ethnic study of atherosclerosis (MESA) study. Circ Cardiovasc Imaging. 2014;7(2):292-302.

63. Donekal S, et al. Inter-study reproducibility of cardiovascular magnetic resonance tagging. J Cardiovasc Magn Reson. 2013;15(1):37. 